\title{
Fixed-Point Theorems for Rational Interpolative-Type Operators with Applications
}

\author{
Muhammad Sarwar $\left(\mathbb{D},{ }^{1}\right.$ Sahibzada Waseem Ahmad $\mathbb{D}^{1},{ }^{1}$ and Thabet Abdeljawad $\mathbb{D}^{2,3,4}$ \\ ${ }^{1}$ Department of Mathematics, University of Malakand, Chakdara, Dir(L), Khyber Pakhtunkhwa, Pakistan \\ ${ }^{2}$ Prince Sultan University, P.O. Box 66833, Riyadh 11586, Saudi Arabia \\ ${ }^{3}$ Department of Medical Research, China Medical University, Taichung 40402, Taiwan \\ ${ }^{4}$ Department of Computer Science and Information Engineering, Asia University, Taichung, Taiwan
}

Correspondence should be addressed to Muhammad Sarwar; sarwarswati@gmail.com and Thabet Abdeljawad; tabdeljawad@psu.edu.sa

Received 24 June 2020; Revised 14 October 2020; Accepted 8 November 2020; Published 25 November 2020

Academic Editor: Antonio Francisco Roldan Lopez de Hierro

Copyright (c) 2020 Muhammad Sarwar et al. This is an open access article distributed under the Creative Commons Attribution License, which permits unrestricted use, distribution, and reproduction in any medium, provided the original work is properly cited.

In the current manuscript, two fixed-point theorems for Dass-Gupta and Gupta-Saxena rational interpolative-type operators are studied in the setting of metric spaces. For the authenticity of the presented work, examples and applications to the existence of a solution to the Caputo-Fabrizio fractional derivative and Caputo-Fabrizio fractal-fractional derivative are also discussed.

\section{Introduction and Preliminaries}

In [1], Banach proposed his famous postulation, the Banach contraction principle. Banach proposed that a continuous self-operator in the setting of complete metric space possesses a unique fixed point. The contraction condition was generalized in several directions. One of the generalizations was supposed by Kannan [2,3] in which he dropped the continuity assumption of the operator. As a part of the generalization of the Banach contraction principle, Dass-Gupta [4] and Gupta-Saxena [5] introduced the innovation of rational contraction. In recent times, Karapinar [6] converted the classical Kannan [2] contraction to an interpolative Kannan contraction in order to maximize the rate of convergence of an operator to a unique fixed point. However, Karapinar and Agarwal [7] found a little gap in the article [6] about the assumption of the fixed point being unique. They provided a counterexample to verify that the fixed point need not be unique and invalidate the assumption of a unique fixed point.

Invented more than a century ago, fractional calculus has attracted numerous physicists, mathematicians, engineers, and researchers in the field of biological sciences because of its extraordinary involvement in these fields of sciences. Despite the long debate, fractional calculus is still not mature enough, and scientists from every field need to do a lot more in this particular branch of mathematics to solve some of its complicated problems. Fractional calculus is mainly composed of fractional integral equation, fractional differential equations, and fractional integrodifferential equations. From the very first day, scientists are finding ways to solve these types of integral equations cited in the manuscripts which can provide enough insight on these problems and their solution [8-12]; among all the other techniques, one technique is a fixed-point theory; several articles can be found on this topic (see, for instance, [13-17] and references therein).

In the fixed-point theory, researchers mostly try to check the existence of a solution to the problem in an underlying set. Because of its complicated nature, it is sometimes impossible to find the exact solution to fractional-type equations in some cases; therefore, frequently, scientists attempt to find the nature of the solution rather than an analytic or exact solution. The particular type of differential equations, namely, Caputo-Fabrizio fractional and Caputo-Fabrizio fractal-fractional differential equations [18-20], can also be used in many problems of the aforesaid fields like heat 
transfer problem, Fisher reaction diffusion equation, mass spring damper system, modeling of steady heat flow, etc.

Motivated by the work done in $[6,7,21]$, we have converted the famous rational contractions in $[4,5]$ to interpolation rational contractions with examples given in each case to verify each theorem. Consequently, the enormous amount of applications of fractional differential equation has led us to contribute the existence of a solution to a couple of fractional differential equations, i.e., the Caputo-Fabrizio fractional differential equation and Caputo-Fabrizio fractal-fractional differential equation. The analysis in the current study indicates that whenever the aforementioned fractional differential equations satisfy certain conditions under specific circumstances, then a contraction theorem guarantees the existence of at least one solution.

Theorem 1 (see [1]). Consider (M, $\mathfrak{M})$ to be a complete metric space. Let $\mathfrak{T}: \mathfrak{M} \longrightarrow \mathfrak{M}$ be a continuous self-operator if there exists a constant $\delta \in[0,1)$, such that

$$
\mathfrak{p}(\mathfrak{I} \mathfrak{f}, \mathfrak{I} \mathfrak{g}) \leq \delta \mathfrak{p}(\mathfrak{F}, \mathfrak{g})
$$

$\forall \mathfrak{f}, \mathfrak{g} \in \mathfrak{M}$, then $\mathfrak{I}$ possesses a unique fixed point.

Afterwards, a question was raised: what happens to the fixed point when the operator is not continuous? Ultimately, the answer to the question was given by Kannan in [2]. He proposed the following theorem as a modified version of Theorem 1.

Theorem 2 (see[2]). Consider ( $\mathfrak{M}, \mathfrak{p})$ to be a complete metric space. Let $\mathfrak{I}: \mathfrak{M} \longrightarrow \mathfrak{M}$ be as a self-operator if there exists a constant $\delta \in[0,1 / 2)$, such that

$$
\mathfrak{p}(\mathfrak{T} \mathfrak{f}, \mathfrak{T} \mathfrak{g}) \leq \delta[\mathfrak{p}(\mathfrak{f}, \mathfrak{I} \mathfrak{f})+\mathfrak{p}(\mathfrak{g}, \mathfrak{I} \mathfrak{g})]
$$

$\forall \mathfrak{f}, \mathfrak{g} \in \mathfrak{M}$, then $\mathfrak{I}$ possesses a unique fixed point.

Eventually, Theorem 1 was generalized to a rational-type contraction by Gupta et al. $[4,5]$ as follows:

Theorem 3 (see [4]). Consider (M, $\mathfrak{p}$ ) to be a complete metric space. Let $\mathfrak{I}: \mathfrak{M} \longrightarrow \mathfrak{M}$ be a continuous self-operator if there exists constants $\delta_{1}, \delta_{2}>0$, such that $\delta_{1}+\delta_{2}<1$ and

$$
\mathfrak{p}(\mathfrak{T} \mathfrak{F}, \mathfrak{I} \mathfrak{g}) \leq \delta_{1} \frac{\mathfrak{p}(\mathfrak{g}, \mathfrak{T} \mathfrak{g})[1+\mathfrak{p}(\mathfrak{f}, \mathfrak{T} \mathfrak{f})]}{1+\mathfrak{p}(\mathfrak{f}, \mathfrak{g})}+\delta_{2} \mathfrak{p}(\mathfrak{f}, \mathfrak{g})
$$

$\forall \mathfrak{f}, \mathfrak{g} \in \mathfrak{M}$, then $\mathfrak{T}$ possesses a unique fixed point.

Theorem 4 (see [5]). Consider (M, $\mathfrak{M})$ to be a complete metric space. Let $\mathfrak{T}: \mathfrak{M} \longrightarrow \mathfrak{M}$ be a self-operator if there exists constants $\delta_{1}, \delta_{2}, \delta_{3}>0$, such that $\delta_{1}+\delta_{2}+\delta_{3}<1$ and

$$
\begin{aligned}
\mathfrak{p}(\mathfrak{I} \mathfrak{f}, \mathfrak{I} \mathfrak{g}) \leq & \delta_{1} \frac{\mathfrak{p}(\mathfrak{g}, \mathfrak{I} \mathfrak{g})[1+\mathfrak{p}(\mathfrak{f}, \mathfrak{T} \mathfrak{f})]}{1+\mathfrak{p}(\mathfrak{f}, \mathfrak{g})}+\delta_{2} \frac{\mathfrak{p}(\mathfrak{g}, \mathfrak{T} \mathfrak{g}) \mathfrak{p}(\mathfrak{f}, \mathfrak{T} \mathfrak{f})}{1+\mathfrak{p}(\mathfrak{f}, \mathfrak{g})} \\
& +\delta_{3} \mathfrak{p}(\mathfrak{f}, \mathfrak{g}) .
\end{aligned}
$$

$\forall$ distinct $\mathfrak{f}, \mathfrak{g} \in \mathfrak{M}$, then $\mathfrak{I}$ possesses a unique fixed point.

\section{Main Results}

This section provides the extension of the famous rationaltype contractions to interpolative rational contraction.

Before proceeding to the first result of the current section, consider the definition which can later be used in the proof of the first main result of this section.

Definition 5. Suppose ( $\mathfrak{M}, \mathfrak{p})$ be a metric space. Furthermore, consider a continuous operator $\mathfrak{T}: \mathfrak{M} \longrightarrow \mathfrak{M}$. If $\exists \alpha \in(0,1)$ and $\delta \in(0,1)$, such that

$$
\mathfrak{p}(\mathfrak{T} \mathfrak{f}, \mathfrak{T} \mathfrak{g}) \leq \delta\left[\frac{\mathfrak{p}(\mathfrak{g}, \mathfrak{T} \mathfrak{g}) \mathfrak{p}(\mathfrak{f}, \mathfrak{T} \mathfrak{f})}{\mathfrak{p}(\mathfrak{f}, \mathfrak{g})}\right]^{\alpha}[\mathfrak{p}(\mathfrak{f}, \mathfrak{g})]^{1-\alpha}
$$

$\forall$ distinct $\mathfrak{f}, \mathfrak{g} \in \mathfrak{M} / \operatorname{Fix}(\mathfrak{M})$. Then, $\mathfrak{I}$ is known as an interpolative Dass and Gupta rational-type contraction.

It can be analyzed that Definition 5 is the conversion of the Dass-Gupta rational contraction in Theorem 3 to an interpolation Dass-Gupta rational contraction.

Theorem 6. In the setting of a complete metric space, the operator for interpolative Dass and Gupta rational-type contraction defined in Definition 5 possesses a fixed point.

Proof. Taking an arbitrary $\mathfrak{f}_{0} \in(\mathfrak{M}, \mathfrak{p})$ and constructing an iterative sequence $\left\{\tilde{\mathfrak{f}}_{n}\right\}_{n \in \mathbb{N}}$ as $\mathfrak{f}_{n}=\mathfrak{T}\left(\mathfrak{f}_{n-1}\right)=\mathfrak{T}^{n}\left(\mathfrak{f}_{0}\right)$. If $\mathfrak{f}_{n_{0}}=$ $\mathfrak{f}_{n_{0}+1}$ for any $n_{0}>0$, then $\mathfrak{f}_{n_{0}}$ is a fixed point for $\mathfrak{I}$, which completes the proof. Consequently, taking $\mathfrak{f}_{n} \neq \mathfrak{f}_{n+1}$ for each $n \geq 0$ and by replacing $\mathfrak{f}$ by $\mathfrak{f}_{n}$ and $\mathfrak{g}$ by $\mathfrak{f}_{n-1}$ in (5), it is deduced that

$$
\begin{aligned}
& \mathfrak{p}\left(\mathfrak{f}_{n+1}, \mathfrak{f}_{n}\right)=\mathfrak{p}\left(\mathfrak{T} \mathfrak{f}_{n}, \mathfrak{I} \mathfrak{f}_{n-1}\right) \\
& \leq \delta\left[\frac{\mathfrak{p}\left(\mathfrak{f}_{n-1}, \mathfrak{T} \mathfrak{f}_{n-1}\right) \mathfrak{p}\left(\mathfrak{f}_{n}, \mathfrak{T} \mathfrak{f}_{n}\right)}{\mathfrak{p}\left(\mathfrak{f}_{n}, \mathfrak{f}_{n-1}\right)}\right]^{\alpha}\left[\mathfrak{p}\left(\mathfrak{f}_{n}, \tilde{\mathfrak{f}}_{n-1}\right)\right]^{1-\alpha} \\
& =\delta\left[\frac{\mathfrak{p}\left(\mathfrak{f}_{n-1}, \mathfrak{f}_{n}\right) \mathfrak{p}\left(\mathfrak{f}_{n}, \mathfrak{f}_{n+1}\right)}{\mathfrak{p}\left(\tilde{\mathfrak{f}}_{n}, \mathfrak{f}_{n-1}\right)}\right]^{\alpha}\left[\mathfrak{p}\left(\mathfrak{f}_{n}, \tilde{\mathfrak{f}}_{n-1}\right)\right]^{1-\alpha} \\
& =\delta\left[\mathfrak{p}\left(\mathfrak{f}_{n}, \mathfrak{f}_{n+1}\right)\right]^{\alpha}\left[\mathfrak{p}\left(\mathfrak{f}_{n}, \mathfrak{f}_{n-1}\right)\right]^{1-\alpha} \text {. }
\end{aligned}
$$

With straightforward calculation, it can be analyzed that

$$
\left[\mathfrak{p}\left(\mathfrak{f}_{n}, \mathfrak{f}_{n+1}\right)\right]^{1-\alpha} \leq \delta\left[\mathfrak{p}\left(\mathfrak{f}_{n}, \mathfrak{f}_{n-1}\right)\right]^{1-\alpha}
$$

Hence, it can be observed that the sequence $\left\{\mathfrak{p}\left(\mathfrak{f}_{n}, \mathfrak{f}_{n-1}\right)\right\}$ is a sequence of nonnegative terms which is nonincreasing. As a consequence, there is a nonnegative constant $\mathbb{Q}$ such 
that $\lim _{n \rightarrow \infty} \mathfrak{p}\left(\mathfrak{f}_{n}, \mathfrak{f}_{n-1}\right)=\mathfrak{Q}$. It is presumed that $\mathfrak{Q}>0$. Indeed, from (7), it is obvious that

$$
\mathfrak{p}\left(\mathfrak{f}_{n}, \mathfrak{f}_{n+1}\right) \leq \delta \mathfrak{p}\left(\mathfrak{f}_{n}, \mathfrak{f}_{n-1}\right) \leq \cdots \leq \delta^{n} \mathfrak{p}\left(\mathfrak{f}_{0}, \mathfrak{f}_{1}\right)
$$

Letting $n \longrightarrow \infty$ in (8), it can be concluded that $\mathbb{Q}=0$.

As a proceeding step, it is proven that the given sequence is a Cauchy sequence. Using the triangle inequality

$$
\begin{aligned}
\mathfrak{p}\left(\mathfrak{f}_{n}, \mathfrak{f}_{n+m}\right) & \leq \delta \mathfrak{p}\left(\mathfrak{f}_{n}, \mathfrak{f}_{n+1}\right)+\cdots+\delta \mathfrak{p}\left(\mathfrak{f}_{n+m-1}, \mathfrak{f}_{n+m}\right) \\
& \leq \delta^{n} \mathfrak{p}\left(\mathfrak{f}_{0}, \mathfrak{f}_{1}\right)+\cdots+\delta^{n+m-1} \mathfrak{p}\left(\mathfrak{f}_{0}, \mathfrak{f}_{1}\right) \leq \frac{\delta^{n}}{1-\delta} \mathfrak{p}\left(\mathfrak{f}_{0}, \mathfrak{f}_{1}\right)
\end{aligned}
$$

Letting $n \longrightarrow \infty$ in (9), it is deduced that the sequence $\left\{\mathfrak{f}_{n}\right\}_{n \in \mathbb{N}}$ is a Cauchy sequence. As stated, $(\mathfrak{M}, \mathfrak{p})$ is a complete metric space; such an assumption guarantees the existence of a number $\mathfrak{f} \in \mathfrak{M}$ such that $\lim _{n \rightarrow \infty} \mathfrak{p}\left(\mathfrak{f}_{n}, \mathfrak{f}\right)=0$. At last, it is proven that $\mathfrak{f}$ is the fixed point of $\mathfrak{I}$ of the sequence $\left\{\mathfrak{f}_{\mathfrak{n}}\right\}_{n \in \mathbb{N}}$. Suppose that $\mathfrak{f} \neq \mathfrak{T} \mathfrak{F}$; therefore, $\mathfrak{p}(\mathfrak{F}, \mathfrak{I} \mathfrak{F})>0$. Recall that $\mathfrak{f} \neq \mathfrak{T} \mathfrak{F}$ and for each $n \geq 0$ and by letting $\mathfrak{f}=\mathfrak{f}_{n}$ and $\mathfrak{g}=\mathfrak{F}$ in (5), it is determined that

$$
\begin{aligned}
\mathfrak{p}\left(\mathfrak{f}_{n+1}, \mathfrak{T} \mathfrak{f}\right) & =\mathfrak{p}\left(\mathfrak{T} \mathfrak{f}_{n}, \mathfrak{T} \mathfrak{f}\right) \leq \delta\left[\frac{\mathfrak{p}(\mathfrak{f}, \mathfrak{T} \mathfrak{f}) \mathfrak{p}\left(\mathfrak{f}_{n}, \mathfrak{T} \mathfrak{f}_{n}\right)}{\mathfrak{p}\left(\mathfrak{f}_{n}, \mathfrak{f}\right)}\right]^{\alpha}\left[\mathfrak{p}\left(\mathfrak{f}_{n}, \mathfrak{f}\right)\right]^{1-\alpha} \\
& =\delta\left[\frac{\mathfrak{p}(\mathfrak{f}, \mathfrak{T} \mathfrak{f}) \mathfrak{p}\left(\mathfrak{f}_{n}, \mathfrak{f}_{n+1}\right)}{\mathfrak{p}\left(\mathfrak{f}_{n}, \mathfrak{f}\right)}\right]^{\alpha}\left[\mathfrak{p}\left(\mathfrak{f}_{n}, \mathfrak{f}\right)\right]^{1-\alpha}
\end{aligned}
$$

By letting $n \longrightarrow \infty$ in $((10))$, it is analyzed thatp $(\mathfrak{T} \mathfrak{f}, \mathfrak{f})=0$; thus, $\mathfrak{f}=\mathfrak{I}$ fwhich is, subsequently, a contradiction. Therefore, $\mathfrak{f}=\mathfrak{T} \mathfrak{f}$.

The proof is complete.

Example 7. Consider a nonempty set $\mathfrak{M}=\{0,1,2,3\}$ and a distance function $\mathfrak{p}: \mathfrak{M} \times \mathfrak{M} \longrightarrow[0, \infty)$ defined by $\mathfrak{p}=\mid \mathfrak{f}-\mathfrak{g}$ $\mid \forall \mathfrak{f}, \mathfrak{g} \in \mathfrak{M}$. It can be analyzed that the metric space ( $\mathfrak{M}, p)$ is a complete metric space. Next, an operator $\mathfrak{I}: \mathfrak{M} \longrightarrow \mathfrak{M}$, is defined by

$$
\mathfrak{T} \mathfrak{f}= \begin{cases}1, & \text { if } \mathfrak{f} \in\{0,1,3\} \\ 2, & \text { if } \mathfrak{f} \in\{2\}\end{cases}
$$

Now, to check if $\mathfrak{T}$ is a Dass and Gupta-type contraction. Consider, for $\mathfrak{f}=1, \mathfrak{g}=2, \delta_{1}=0.7$, and $\delta_{2}=0.1$

$$
\begin{aligned}
1 & =\mathfrak{p}(\mathfrak{I} 1, \mathfrak{T} 2)>\delta_{1}\left[\frac{\mathfrak{p}(\mathfrak{g}, \mathfrak{T} \mathfrak{g})[1+\mathfrak{p}(\mathfrak{f}, \mathfrak{T} \mathfrak{f})]}{1+\mathfrak{p}(\mathfrak{f}, \mathfrak{g})}\right]+\delta_{2}[\mathfrak{p}(\mathfrak{f}, \mathfrak{g})] \\
& =\delta_{1}\left[\frac{\mathfrak{p}(2,2)[1+\mathfrak{p}(1,1)]}{1+\mathfrak{p}(1,2)}\right]+\delta_{2}[\mathfrak{p}(1,2)]=\delta_{2} .
\end{aligned}
$$

As $\delta_{1}, \delta_{2} \in(0,1)$ along with $\delta_{1}+\delta_{2}=0.8<1$, so that $\mathfrak{I}$ does not satisfy the inequality (3). Therefore, it is not a Dass-Gupta-type contraction in Theorem 3.
On the contrary for $\delta=0.15$ and $\alpha=1 / 2$. Let $\mathfrak{f}, \mathfrak{g} \in \mathfrak{M} /$ $\operatorname{Fix}(\mathfrak{M})$, then $\mathfrak{f}, \mathfrak{g} \in\{(0,3),(3,0)\}$, i.e., two cases arise in general:

Case 1. If $\mathfrak{f}=0$ and $\mathfrak{g}=3$, then

$$
0=\mathfrak{p}(\mathfrak{T} 0, \mathfrak{I} 3)<\delta\left[\frac{\mathfrak{p}(3, \mathfrak{T} 3) \mathfrak{p}(0, \mathfrak{T} 0)}{\mathfrak{p}(0,3)}\right]^{\alpha}[\mathfrak{p}(0,3)]^{1-\alpha}=0.212
$$

Case 2. If $\mathfrak{f}=3$ and $\mathfrak{g}=0$, then

$$
0=\mathfrak{p}(\mathfrak{T} 3, \mathfrak{T} 0)<\delta\left[\frac{\mathfrak{p}(0, \mathfrak{I} 0) \mathfrak{p}(3, \mathfrak{I} 3)}{\mathfrak{p}(3,0)}\right]^{\alpha}[\mathfrak{p}(3,0)]^{1-\alpha}=0.212
$$

Henceforth, the self-operator $\mathfrak{I}$ is an interpolative Dass and Gupta-type contraction with the data provided above. The fixed points are 1,2.

Next, consider the extension of the Gupta-Saxena-type rational contraction to interpolative contraction. Before proceeding to the theorem, consider the definition.

Definition 8. Consider ( $\mathfrak{M}, \mathfrak{p})$ to be a metric space. In addition, consider a continuous operator $\mathfrak{T}: \mathfrak{M} \longrightarrow \mathfrak{M}$. If $\exists$ $\alpha, \beta \in(0,1)$ and $\delta \in(0,1)$, such that

$\mathfrak{p}(\mathfrak{T} \mathfrak{f}, \mathfrak{T} \mathfrak{g}) \leq \delta\left[\frac{\mathfrak{p}(\mathfrak{f}, \mathfrak{T} \mathfrak{f}) \mathfrak{p}(\mathfrak{g}, \mathfrak{T} \mathfrak{g})}{\mathfrak{p}(\mathfrak{f}, \mathfrak{g})}\right]^{\alpha}\left[\frac{\mathfrak{p}(\mathfrak{g}, \mathfrak{T} \mathfrak{g}) \mathfrak{p}(\mathfrak{f}, \mathfrak{T} \mathfrak{f})}{\mathfrak{p}(\mathfrak{f}, \mathfrak{g})}\right]^{\beta}[\mathfrak{p}(\mathfrak{f}, \mathfrak{g})]^{1-\alpha-\beta}$

$\forall$ distinct $\mathfrak{f}, \mathfrak{g} \in \mathfrak{M} / \operatorname{Fix}(\mathfrak{M})$. Then, $\mathfrak{T}$ is known as an interpolative Gupta-Saxena rational-type contraction.

It can be analyzed that Definition 8 is the conversion of Gupta-Saxena rational contraction in Theorem 4 to an interpolation Gupta-Saxena rational contraction.

Theorem 9. In the setting of a complete metric space, the operator for interpolative Gupta-Saxena rational-type contraction defined in Definition 8 possesses a fixed point.

Proof. By taking an arbitrary $\mathfrak{f}_{0} \in(\mathfrak{M}, \mathfrak{p})$ and constructing an iterative sequence $\left\{\mathfrak{f}_{n}\right\}_{n \in \mathbb{N}}$ as $\mathfrak{f}_{n}=\mathfrak{T}\left(\mathfrak{f}_{n-1}\right)=\mathfrak{T}^{n}\left(\mathfrak{f}_{0}\right)$. If $\mathfrak{f}_{n_{0}}=$ $\mathfrak{f}_{n_{0}+1}$ for any $n_{0}>0$, then $\mathfrak{f}_{0}$ is a fixed point for $\mathfrak{T}$, which completes the proof. Consequently, taking $\mathfrak{f}_{n} \neq \mathfrak{f}_{n+1}$ for each $n \geq 0$ and by replacing $\mathfrak{f}$ by $\mathfrak{f}_{n}$ and $\mathfrak{g}$ by $\mathfrak{f}_{n-1}$ in (15), it is deduced that 


$$
\begin{aligned}
& \mathfrak{p}\left(\mathfrak{f}_{n+1}, \mathfrak{f}_{n}\right)=\mathfrak{p}\left(\mathfrak{T} \mathfrak{f}_{n}, \mathfrak{I} \mathfrak{f}_{n-1}\right) \leq \delta\left[\frac{\mathfrak{p}\left(\mathfrak{f}_{n}, \mathfrak{T} \mathfrak{f}_{n}\right) \mathfrak{p}\left(\mathfrak{f}_{n-1}, \mathfrak{T} \mathfrak{f}_{n-1}\right)}{\mathfrak{p}\left(\mathfrak{f}_{n}, \mathfrak{f}_{n-1}\right)}\right]^{\alpha} \\
& \cdot\left[\frac{\mathfrak{p}\left(\mathfrak{f}_{n-1}, \mathfrak{T} \mathfrak{f}_{n-1}\right) \mathfrak{p}\left(\mathfrak{f}_{n}, \mathfrak{T} \mathfrak{f}_{n}\right)}{\mathfrak{p}\left(\mathfrak{f}_{n}, \mathfrak{f}_{n-1}\right)}\right]^{\beta}\left[\mathfrak{p}\left(\mathfrak{f}_{n}, \mathfrak{f}_{n-1}\right)\right]^{1-\alpha-\beta} \\
& =\delta\left[\frac{\mathfrak{p}\left(\mathfrak{f}_{n}, \mathfrak{f}_{n+1}\right) \mathfrak{p}\left(\mathfrak{f}_{n-1}, \mathfrak{f}_{n}\right)}{\mathfrak{p}\left(\tilde{\mathfrak{f}}_{n}, \mathfrak{f}_{n-1}\right)}\right]^{\alpha}\left[\frac{\mathfrak{p}\left(\mathfrak{f}_{n-1}, \mathfrak{f}_{n}\right) \mathfrak{p}\left(\mathfrak{f}_{n}, \mathfrak{f}_{n+1}\right)}{\mathfrak{p}\left(\tilde{\mathfrak{f}}_{n}, \mathfrak{f}_{n-1}\right)}\right]^{\beta} \\
& \cdot\left[\mathfrak{p}\left(\mathfrak{f}_{n}, \mathfrak{f}_{n-1}\right)\right]^{1-\alpha-\beta}=\delta\left[\mathfrak{p}\left(\mathfrak{f}_{n}, \mathfrak{f}_{n+1}\right)\right]^{\alpha+\beta}\left[\mathfrak{p}\left(\mathfrak{f}_{n}, \mathfrak{f}_{n-1}\right)\right]^{1-\alpha-\beta} \text {. }
\end{aligned}
$$

With straightforward calculation, it can be analyzed that

$$
\left[\mathfrak{p}\left(\mathfrak{f}_{n}, \mathfrak{f}_{n+1}\right)\right]^{1-\alpha-\beta} \leq \delta\left[\mathfrak{p}\left(\mathfrak{f}_{n}, \mathfrak{f}_{n-1}\right)\right]^{1-\alpha-\beta}
$$

Hence, it can be observed that the sequence $\left\{\mathfrak{p}\left(\tilde{\mathfrak{f}}_{n}, \mathfrak{f}_{n-1}\right)\right\}$ is a sequence of nonnegative terms which is nonincreasing. As a consequence, there is a nonnegative constant $\mathbb{Q}$ such that $\lim _{n \rightarrow \infty} \mathfrak{p}\left(\tilde{\mathfrak{f}}_{n}, \tilde{\mathfrak{f}}_{n-1}\right)=\mathfrak{Q}$. It is presumed that $\mathfrak{Q}>0$. Indeed, from (17), it is obvious that

$$
\mathfrak{p}\left(\mathfrak{f}_{n}, \mathfrak{f}_{n+1}\right) \leq \delta \mathfrak{p}\left(\mathfrak{f}_{n}, \mathfrak{f}_{n-1}\right) \leq \cdots \leq \delta^{n} \mathfrak{p}\left(\mathfrak{f}_{0}, \mathfrak{f}_{1}\right)
$$

Letting $n \longrightarrow \infty$ in (18), it can be concluded that $\mathbb{R}=0$.

As a proceeding step, it is proven that the given sequence is a Cauchy sequence. Using the triangle inequality

$$
\begin{aligned}
\mathfrak{p}\left(\mathfrak{f}_{n}, \mathfrak{f}_{n+m}\right) & \leq \delta \mathfrak{p}\left(\mathfrak{f}_{n}, \mathfrak{f}_{n+1}\right)+\cdots+\delta \mathfrak{p}\left(\mathfrak{f}_{n+m-1}, \mathfrak{f}_{n+m}\right) \\
& \leq \delta^{n} \mathfrak{p}\left(\mathfrak{f}_{0}, \mathfrak{f}_{1}\right)+\cdots+\delta^{n+m-1} \mathfrak{p}\left(\mathfrak{f}_{0}, \tilde{\mathfrak{f}}_{1}\right) \leq \frac{\delta^{n}}{1-\delta} \mathfrak{p}\left(\mathfrak{\mathfrak { f }}_{0}, \mathfrak{\mathfrak { f }}_{1}\right)
\end{aligned}
$$

Letting $n \longrightarrow \infty$ in (19), it is deduced that the sequence $\left\{\mathfrak{f}_{n}\right\}_{n \in \mathbb{N}}$ is a Cauchy sequence. As stated, $(\mathfrak{M}, \mathfrak{p})$ is a complete metric space; such an assumption guarantees the existence of a number $\mathfrak{f}_{0} \in \mathfrak{M}$ such that $\lim _{n \rightarrow \infty} \mathfrak{p}\left(\mathfrak{f}_{n}, \mathfrak{f}_{0}\right)=0$. At last, it is proven that $\mathfrak{f}_{0}$ is the fixed point of $\mathfrak{I}$ of the sequence $\left\{\mathfrak{f}_{\mathfrak{n}}\right\}_{n \in \mathbb{N}}$. Suppose that $\mathfrak{f} \neq \mathfrak{T} \mathfrak{F}$; therefore, $\mathfrak{p}(\mathfrak{f}, \mathfrak{I} \mathfrak{f})>0$. Recall that $\mathfrak{f} \neq \mathfrak{T} \mathfrak{f}$ and for each $n \geq 0$ and by letting $\mathfrak{f}=\mathfrak{f}_{n}$ and $\mathfrak{g}=\mathfrak{f}$ in (15), it is determined that

$$
\begin{aligned}
\mathfrak{p}\left(\mathfrak{f}_{n+1}, \mathfrak{I} \mathfrak{\mathfrak { f }}\right)= & \mathfrak{p}\left(\mathfrak{T} \mathfrak{f}_{n}, \mathfrak{T} \mathfrak{f}\right) \leq \delta\left[\frac{\mathfrak{p}\left(\mathfrak{f}_{n}, \mathfrak{T} \mathfrak{f}_{n}\right) \mathfrak{p}(\mathfrak{f}, \mathfrak{T} \mathfrak{f})}{\mathfrak{p}\left(\mathfrak{f}_{n}, \mathfrak{f}\right)}\right]^{\alpha} \\
& \cdot\left[\frac{\mathfrak{p}(\mathfrak{f}, \mathfrak{T} \mathfrak{f}) \mathfrak{p}\left(\mathfrak{f}_{n}, \mathfrak{T} \mathfrak{f}_{n}\right)}{\mathfrak{p}\left(\mathfrak{f}_{n}, \mathfrak{f}\right)}\right]^{\beta}\left[\mathfrak{p}\left(\mathfrak{f}_{n}, \mathfrak{f}\right)\right]^{1-\alpha-\beta} \\
= & \delta\left[\frac{\mathfrak{p}\left(\mathfrak{f}_{n}, \mathfrak{f}_{n+1}\right) \mathfrak{p}(\mathfrak{f}, \mathfrak{T} \mathfrak{f})}{\mathfrak{p}\left(\tilde{f}_{n}, \mathfrak{f}\right)}\right]^{\alpha}\left[\frac{\mathfrak{p}(\mathfrak{f}, \mathfrak{T} \mathfrak{f}) \mathfrak{p}\left(\mathfrak{f}_{n}, \mathfrak{f}_{n+1}\right)}{\mathfrak{p}\left(\mathfrak{f}_{n}, \mathfrak{f}\right)}\right]^{\beta} \\
& \cdot\left[\mathfrak{p}\left(\mathfrak{f}_{n}, \mathfrak{f}\right)\right]^{1-\alpha-\beta} .
\end{aligned}
$$

By letting $n \longrightarrow \infty$ in $(20)$, it is analyzed that $\mathfrak{p}\left(\mathfrak{I}_{\mathfrak{n}}\right.$, $\mathfrak{f})=0$; thus, $\mathfrak{f}=\mathfrak{T} \mathfrak{f}$, which is, subsequently, a contradiction. Therefore, $\mathfrak{f}=\mathfrak{T} \mathfrak{f}$.

The proof is complete.
Example 10. Consider the distances and operator defined in Example 7. In the proceeding example, it is shown that $\mathfrak{T}$ is not a Gupta-Saxena-type contraction for $\delta_{1}=0.3, \delta_{2}=0.1$, and $\delta_{3}=0.4$. However, $\mathfrak{T}$ is an interpolative version of the Gupta-Saxena-type contraction, i.e., for $\mathfrak{f}=0$ and $\mathfrak{g}=2$

$$
\begin{aligned}
1= & \mathfrak{p}(\mathfrak{T} 1, \mathfrak{T} 2)>\delta_{1}\left[\frac{\mathfrak{p}(\mathfrak{g}, \mathfrak{T} \mathfrak{g})[1+\mathfrak{p}(\mathfrak{f}, \mathfrak{T} \mathfrak{f})]}{1+\mathfrak{p}(\mathfrak{f}, \mathfrak{g})}\right]+\delta_{2} \frac{\mathfrak{p}(\mathfrak{g}, \mathfrak{T} \mathfrak{g}) \mathfrak{p}(\mathfrak{f}, \mathfrak{T} \mathfrak{f})}{1+\mathfrak{p}(\mathfrak{\mathfrak { f }}, \mathfrak{g})} \\
& +\delta_{3}[\mathfrak{p}(\mathfrak{f}, \mathfrak{g})]=\delta_{1}\left[\frac{\mathfrak{p}(2,2)[1+\mathfrak{p}(0,1)]}{1+\mathfrak{p}(0,2)}\right]+\delta_{2} \frac{\mathfrak{p}(2,2) \mathfrak{p}(0,1)}{1+\mathfrak{p}(0,2)} \\
& +\delta_{3}[\mathfrak{p}(0,2)]=2 \delta_{3} .
\end{aligned}
$$

Since $\delta_{1}, \delta_{2}, \delta_{3} \in(0,1)$ along with $\delta_{1}+\delta_{2}+\delta_{3}<1$ suggests that $\mathfrak{I}$ does not satisfy inequality (4); therefore, it is not a Gupta-Saxena-type contraction in Theorem 4.

On the contrary, for $\delta=0.15, \alpha=1 / 3$ and $\beta=1 / 3$. Let $\mathfrak{f}$, $\mathfrak{g} \in \mathfrak{M} / \operatorname{Fix}(\mathfrak{M})$, then $\mathfrak{f}, \mathfrak{g} \in\{(0,3),(3,0)\}$, i.e., two cases arise in general:

Case 1. If $\mathfrak{f}=0$ and $\mathfrak{g}=3$, then

$$
\begin{aligned}
0= & \mathfrak{p}(\mathfrak{T} 0, \mathfrak{I} 3)<\delta\left[\frac{\mathfrak{p}(0, \mathfrak{I} 0) \mathfrak{p}(3, \mathfrak{I} 3)}{\mathfrak{p}(0,3)}\right]^{\alpha} \\
& \cdot\left[\frac{\mathfrak{p}(3, \mathfrak{I} 3) \mathfrak{p}(0, \mathfrak{I} 0)}{\mathfrak{p}(0,3)}\right]^{\beta}[\mathfrak{p}(0,3)]^{1-\alpha-\beta}=0.13332 .
\end{aligned}
$$

Case 2. If $\mathfrak{f}=3$ and $\mathfrak{g}=0$, then

$$
\begin{aligned}
0= & \mathfrak{p}(\mathfrak{T} 3, \mathfrak{T} 0)<\delta\left[\frac{\mathfrak{p}(3, \mathfrak{T} 3) \mathfrak{p}(0, \mathfrak{T} 0)}{\mathfrak{p}(3,0)}\right]^{\alpha} \\
& \cdot\left[\frac{\mathfrak{p}(0, \mathfrak{T} 0) \mathfrak{p}(3, \mathfrak{I} 3)}{\mathfrak{p}(3,0)}\right]^{\beta}[\mathfrak{p}(3,0)]^{1-\alpha-\beta}=0.1333
\end{aligned}
$$

Henceforth, the self-operator $\mathfrak{T}$ is an interpolative version of the Gupta-Saxena-type contraction with the data provided in Example 7. The fixed points are 1,2.

\section{Applications}

The current section provides the existence of a solution to the Caputo-Fabrizio fractional derivative and the CaputoFabrizio fractal-fractional derivative. The very first result of this section is the existence of the solution to the CaputoFabrizio fractional derivative.

The Caputo-Fabrizio fractional derivative of order $\gamma$ is defined as

$$
{ }^{\mathrm{CF}} D_{t}^{\gamma} \mathfrak{\mathcal { U }}(t)=\frac{N(\gamma)}{1-\gamma} \int_{0}^{t} \mathfrak{\mathfrak { I }}^{\prime}(\tau) \exp \left[-\frac{\gamma}{1-\gamma}(t-\tau)\right] d \tau
$$

with boundary condition $\mathfrak{A}(0)=0$ where $N(\gamma)$ is a normalization function satisfying $N(0)=N(1)=1$ and $a$ $\leq t \leq \tau \leq b$. 
Consider $\mathfrak{M}=C([0,1], \mathfrak{R})$ to be the space of continuous functions on the interval $[0,1]$. Furthermore, consider a distance function $\mathfrak{p}: \mathfrak{M}^{2} \longrightarrow[0, \infty)$ defined by $\mathfrak{p}=|\mathfrak{f}-\mathfrak{g}|$. Henceforth, the metric space $(\mathfrak{M}, \mathfrak{p})$ is complete. Now, consider a self-operator $\mathfrak{I}: \mathfrak{M} \longrightarrow \mathfrak{M}$ defined by

$$
\mathfrak{I} \mathfrak{A}=\frac{N(\gamma)}{1-\gamma} \int_{0}^{t} \mathfrak{A}^{\prime}(\tau) \exp \left[-\frac{\gamma}{1-\gamma}(t-\tau)\right] d \tau
$$

Then, the fractional derivative (24) will have a solution if the following condition is satisfied:

$$
|\mathfrak{A}(t)-\mathfrak{B}(t)| \leq \frac{2-\gamma}{\mathfrak{K}(1-\gamma)} \Pi(\mathfrak{A}, \mathfrak{B}),
$$

for all $t \in[0,1]$ where $\alpha, \delta \in(0,1), \mathfrak{K}>0$, and $\Pi(\mathfrak{A}$, $\mathfrak{B})=\delta[\mathfrak{p}(\mathfrak{B}, \mathfrak{I} \mathfrak{B}) \mathfrak{p}(\mathfrak{A}, \mathfrak{I} \mathfrak{A}) / \mathfrak{p}(\mathfrak{A}, \mathfrak{B})]^{\alpha}[\mathfrak{p}(\mathfrak{A}, \mathfrak{B})]^{1-\alpha}$.

To begin the proof, consider

$$
\begin{aligned}
|\mathfrak{I} \mathfrak{A}-\mathfrak{I} \mathfrak{B}|= & \mid \frac{N(\gamma)}{1-\gamma} \int_{0}^{t} \mathfrak{A}^{\prime}(\tau) \exp \left[-\frac{\gamma}{1-\gamma}(t-\tau)\right] d \tau \\
& -\frac{N(\gamma)}{1-\gamma} \int_{0}^{t} \mathfrak{B}^{\prime}(\tau) \exp \left[-\frac{\gamma}{1-\gamma}(t-\tau)\right] d \tau \mid \\
\leq & \frac{N(\gamma)}{1-\gamma}\left[\exp \left[-\frac{\gamma}{1-\gamma}(t-\tau)\right]|\mathfrak{A}(\tau)|_{0}^{t}-\left.\mathfrak{B}(\tau)\right|_{0} ^{t} \mid\right. \\
& \left.+\frac{\gamma}{1-\gamma} \int_{0}^{t} \exp \left[-\frac{\gamma}{1-\gamma}(t-\tau)\right]|\mathfrak{A}(\tau)-\mathfrak{B}(\tau)| d \tau\right] \\
\leq & \frac{N(\gamma)}{1-\gamma}|\mathfrak{A}(t)-\mathfrak{B}(t)|+\frac{N(\gamma)}{1-\gamma}\left[\exp \left[-\frac{\gamma}{1-\gamma}(t)\right] \mid \mathfrak{A}(0)\right. \\
& -\mathfrak{B}(0)\left|+\frac{\gamma}{(1-\gamma)} \int_{0}^{t} \exp \left[-\frac{\gamma}{1-\gamma}(t-\tau)\right]\right| \mathfrak{A}(\tau) \\
& -\mathfrak{B}(\tau) \mid d \tau] .
\end{aligned}
$$
as

Using inequality (26), the above inequality can be written

$$
\begin{aligned}
|\mathfrak{T} \mathfrak{A}-\mathfrak{I} \mathfrak{B}| & \leq \frac{(2-\gamma) N(\gamma)}{\mathfrak{K}(1-\gamma)} \frac{1}{\mathfrak{K}} \Pi(\mathfrak{A}, \mathfrak{B})+\frac{(2-\gamma) N(\gamma)}{\mathfrak{K}(1-\gamma)} \frac{1}{\Pi}(\mathfrak{A} \\
& =\frac{2(2-\gamma) N(\gamma)}{\mathfrak{K}(1-\gamma)} \Pi(\mathfrak{A}, \mathfrak{B}) .
\end{aligned}
$$

So,

$$
\mathfrak{p}(\mathfrak{T} \mathfrak{A}, \mathfrak{T} \mathfrak{B}) \leq \delta \Pi(\mathfrak{A}, \mathfrak{B}),
$$

where $\delta=2(2-\gamma) N(\gamma) / \kappa(1-\gamma)$. Consequently, all the propositions of Theorem 6 are satisfied. Therefore, the equation (24) possesses a solution.

Next, we discuss the existence of a solution to the Caputo-Fabrizio fractal-fractional derivative
Consider the Caputo-Fabrizio fractal-fractional derivative of order $\gamma$ as

$$
{ }_{a}^{\mathrm{CFFF}} D_{t}^{\gamma} \mathfrak{g}(t)=\frac{1-\gamma}{N(\gamma)} 9 t^{9-1} \mathfrak{g}(t, \mathfrak{y}(t))+\frac{\gamma \vartheta}{N(\gamma)} \int_{0}^{t} \tau^{9-1} \mathfrak{g}(\tau, \mathfrak{y}(\tau)) d \tau,
$$

where $N(\gamma)$ is a normalization function satisfying $N$ $(0)=N(1)=1$.

Consider $\mathfrak{M}=C([0,1], \mathfrak{R})$ to be the space of continuous functions on the interval $[0,1]$. Furthermore, consider a distance function $\mathfrak{p}: \mathfrak{M}^{2} \longrightarrow[0, \infty)$ defined by $\mathfrak{p}=|\mathfrak{f}-\mathfrak{g}|$. Henceforth, the metric space $(\mathfrak{M}, \mathfrak{p})$ is complete. Now, consider a self-operator $\mathfrak{I}: \mathfrak{M} \longrightarrow \mathfrak{M}$ defined by

$$
\mathfrak{I} \mathfrak{g}=\frac{1-\gamma}{N(\gamma)} \vartheta t^{\vartheta-1} \mathfrak{g}(t, \mathfrak{y}(t))+\frac{\gamma \vartheta}{N(\gamma)} \int_{0}^{t} \tau^{\vartheta-1} \mathfrak{g}(\tau, \mathfrak{y}(\tau)) d \tau .
$$

Then, the fractal-fractional derivative (30) will have a solution if the operator (31) satisfies the given conditions $\forall t$ $\in[0,1]$

$$
\begin{array}{r}
\mid \mathfrak{g}_{1}\left(t, \mathfrak{y}(t)-\mathfrak{g}_{2}\left(t, \mathfrak{y}(t) \mid \leq \Xi\left(\mathfrak{g}_{1}, \mathfrak{g}_{2}\right),\right.\right. \\
(1-\gamma) \vartheta-\gamma t \leq \frac{\delta N(\gamma)}{t^{9-1}},
\end{array}
$$

where $\quad \Xi\left(\mathfrak{g}_{1}, \mathfrak{g}_{2}\right)=\left[\mathfrak{p}\left(\mathfrak{g}_{1}, \mathfrak{T} \mathfrak{g}_{1}\right) \mathfrak{p}\left(\mathfrak{g}_{2}, \mathfrak{T} \mathfrak{g}_{2}\right) / \mathfrak{p}\left(\mathfrak{g}_{1}, \mathfrak{g}_{2}\right)\right]^{\alpha}\left[\mathfrak{p}\left(\mathfrak{g}_{2}\right.\right.$, $\left.\left.\mathfrak{T} \mathfrak{g}_{2}\right) \mathfrak{p}\left(\mathfrak{g}_{1}, \mathfrak{T} \mathfrak{g}_{1}\right) / \mathfrak{p}\left(\mathfrak{g}_{1}, \mathfrak{g}_{2}\right)\right]^{\beta}\left[\mathfrak{p}\left(\mathfrak{g}_{1}, \mathfrak{g}_{2}\right)\right]^{1-\alpha-\beta}$ and $\delta, \alpha, \beta \in(0$, $1)$.

Consider,

$$
\begin{aligned}
\left|\mathfrak{I}_{1}-\mathfrak{T} \mathfrak{g}_{2}\right|= & \mid \frac{1-\gamma}{N(\gamma)} 9 t^{\vartheta-1} \mathfrak{g}_{1}(t, \mathfrak{y}(t))+\frac{\gamma \vartheta}{N(\gamma)} \int_{0}^{t} \tau^{\vartheta-1} \mathfrak{g}_{1}(\tau, \mathfrak{y}(\tau)) d \tau \\
& -\frac{1-\gamma}{N(\gamma)} 9 t^{\vartheta-1} \mathfrak{g}_{2}(t, \mathfrak{y}(t))-\frac{\gamma \vartheta}{N(\gamma)} \int_{0}^{t} \tau^{\vartheta-1} \mathfrak{g}_{2}(\tau, \mathfrak{y}(\tau)) d \tau \mid \\
\leq & \frac{1-\gamma}{N(\gamma)} \vartheta t^{\vartheta-1}\left|\mathfrak{g}_{1}(t, \mathfrak{y}(t))-\mathfrak{g}_{2}(t, \mathfrak{h}(t))\right| \\
& +\frac{\gamma \vartheta}{N(\gamma)} \int_{0}^{t} \tau^{\vartheta-1}\left|\mathfrak{g}_{1}(\tau, \mathfrak{y}(\tau))-\mathfrak{g}_{2}(\tau, \mathfrak{h}(\tau))\right| d \tau .
\end{aligned}
$$

With the help of (32), the above inequality can be written as

$$
\begin{aligned}
\left|\mathfrak{I} \mathfrak{g}_{1}-\mathfrak{I} \mathfrak{g}_{2}\right| & \leq \frac{1-\gamma}{N(\gamma)} \vartheta t^{\vartheta-1} \Xi\left(\mathfrak{g}_{1}, \mathfrak{g}_{2}\right)+\frac{\gamma \vartheta}{N(\gamma)} \Xi\left(\mathfrak{g}_{1}, \mathfrak{g}_{2}\right) \int_{0}^{t} \tau^{\vartheta-1} d \tau \\
& =\frac{t^{\vartheta-1}}{N(\gamma)}[(1-\gamma) \vartheta-\gamma t] \Xi\left(\mathfrak{g}_{1}, \mathfrak{g}_{2}\right) .
\end{aligned}
$$

Finally, using (33) in the above inequality,

$$
\mathfrak{p}\left(\mathfrak{T} \mathfrak{g}_{1}, \mathfrak{T} \mathfrak{g}_{2}\right) \leq \delta \Xi\left(\mathfrak{g}_{1}, \mathfrak{g}_{2}\right) .
$$


Subsequently, all the hypotheses of Theorem 9 are satisfied. Therefore, Equation (30) has a solution.

\section{Conclusion}

In this study, we converted the famous Dass and Gupta rational contraction and Gupta-Saxena rational contraction to their respective interpolative versions. The motivation for this work is to obtain the fixed point faster than the classical versions of the aforesaid contractions. Further, we studied the existence of a solution to Caputo-Fabrizio fractional differential equation and Caputo-Fabrizio fractal-fractional derivative utilizing the obtained rational interpolation to validate the results.

\section{Data Availability}

No data were used to support this study.

\section{Conflicts of Interest}

The authors declare that they have no competing interest regarding this manuscript.

\section{Authors' Contributions}

All authors contribute equally to the writing of this manuscript. All authors read and approve the final version.

\section{Acknowledgments}

The third author would like to thank Prince Sultan University for funding this work through the research group Nonlinear Analysis Methods in Applied Mathematics (NAMAM), group number RG-DES-2017-01-17.

\section{References}

[1] S. Banach, "Sur les opérations dans les ensembles abstraits et leur application aux équations intégrales," Fundamenta Mathematicae, vol. 3, pp. 133-181, 1922.

[2] R. Kannan, "Some results on fixed points," Bulletin of the Calcutta Mathematical Society, vol. 60, pp. 71-76, 1968.

[3] R. Kannan, "Some results on fixed point II," The American Mathematical Monthly, vol. 76, pp. 405-408, 1969.

[4] B. K. Dass and S. Gupta, "An extension of Banach contraction principle through rational expression," Indian Journal of Pure and Applied Mathematics, vol. 6, pp. 1455-1458, 1975.

[5] A. N. Gupta and A. Saxena, "A unique fixed point theorem in metric spaces," Matematychni Studii, vol. 52, 1984.

[6] E. Karapinar, "Revisiting the Kannan type contractions via interpolation," Advances in the Theory of Nonlinear Analysis and its Application, vol. 2, no. 2, pp. 85-87, 2018.

[7] E. Karapinar, R. Agarwal, and H. Aydi, "Interpolative Reich-Rus-Ćirić type contractions on partial metric spaces," Mathematics, vol. 6, no. 11, p. 256, 2018.

[8] A. A. Hamoudi, K. P. Ghadle, and S. M. Atshan, "The approximate solutions of fractional integro-differential equations by using Adomian decomposition method," The Khayyam Journal of Mathematics, vol. 5, no. 1, pp. 21-39, 2019.
[9] A. Arikoglu and I. Ozkol, "Solution of fractional differential equations by using differential transform method," Chaos, Solitons \& Fractals, vol. 34, no. 5, pp. 1473-1481, 2007.

[10] E. Ahmed, A. M. A. El-Sayed, and H. A. A. El-Saka, "Equilibrium points, stability and numerical solutions of fractionalorder predator-prey and rabies models," Journal of Mathematical Analysis and Applications, vol. 325, no. 1, pp. 542-553, 2007.

[11] E. A. Rawashdeh, "Numerical solution of fractional integrodifferential equations by collocation method," Applied Mathematics and Computation, vol. 176, no. 1, pp. 1-6, 2006.

[12] M. Muslim, C. Conca, and A. K. Nandakumaran, "Approximation of solutions to fractional integral equation," Computers $\&$ Mathematcs with Applications, vol. 59, no. 3, pp. 1236-1244, 2010.

[13] A. Aghajani, Y. Jalilian, and J. Trujillo, "On the existence of solutions of fractional integro-differential equations," Fractional Calculus and Applied Analysis, vol. 15, no. 1, pp. 4459, 2012.

[14] P. Borisut, P. Kumam, V. Gupta, and N. Mani, "Generalized $(\psi, \alpha, \beta)$-weak contractions for initial value problems," Mathematics, vol. 7, no. 3, p. 266, 2019.

[15] R. Ponce, "Bounded mild solutions to fractional integrodifferential equations in Banach spaces," Semigroup Forum, vol. 87, no. 2, pp. 377-392, 2013.

[16] S. K. Panda, T. Abdeljawad, and C. Ravichandran, "A complex valued approach to the solutions of Riemann-Liouville integral, Atangana-Baleanu integral operator and non-linear telegraph equation via fixed point method," Chaos Solitons and Fractals, vol. 130, article 109439, 2020.

[17] V. Gupta, N. Mani, and N. Sharma, "Fixed-point theorems for weak $(\psi, \beta)$-mappings satisfying generalizedC-condition and its application to boundary value problem," Computational and Mathematical Methods, vol. 1, no. 4, 2019.

[18] A. Atangana and S. İ. Araz, "New numerical method for ordinary differential equations: Newton polynomial," Journal of Computational and Applied Mathematics, vol. 372, article 112622, 2020.

[19] A. Alsaedi, D. Baleanu, S. Etemad, and S. Rezapour, "On coupled systems of time-fractional differential problems by using a new fractional derivative," Journal of Function Spaces, vol. 2016, Article ID 4626940, 8 pages, 2016.

[20] K. Liu, M. Fečkan, D. O’Regan, and J. R. Wang, “Hyers-Ulam Stability and Existence of Solutions for Differential Equations with Caputo-Fabrizio Fractional Derivative," Mathematics, vol. 7, no. 4, p. 333, 2019.

[21] E. Karapinar, O. Alqahtani, and H. Aydi, "On interpolative Hardy-Rogers type contractions,” Symmetry, vol. 11, no. 1, p. $8,2019$. 